\title{
Odd-Even Effects in Ion-Beam-Induced Desorption of Biphenyl-Substituted Alkanethiol Self-Assembled Monolayers
}

\author{
Frederik Vervaecke, ${ }^{[a]}$ Sabina Wyczawska, ${ }^{[a]}$ Piotr Cyganik, ${ }^{*[b]}$ Jeroen Bastiaansen, ${ }^{[a]} Z$ bigniew Postawa, ${ }^{[b]}$ \\ Roger E. Silverans, ${ }^{[a]}$ Erno Vandeweert, ${ }^{[a]}$ and Peter Lievens ${ }^{*[a]}$
}

Due to the ease of their preparation and their relatively high stability, self-assembled monolayers (SAMs) are promising candidates to be used in the development of micro- and nanostructured materials with various functionalities. ${ }^{[1]}$ The formation of SAMs is mainly driven by a combination of moleculesubstrate and intermolecular interactions. So far, most of the fundamental studies of SAMs structures have been performed on simple alkanethiols chemisorbed on the $A u(111)$ substrate. ${ }^{[1,2]}$ The importance of alkane length odd-even effects in SAMs has been recently reviewed. ${ }^{[3]}$ In particular, the odd-even effect was observed in the reaction of low-energy pyrazine and $\left[D_{6}\right]$ benzene molecular ions with the terminal group (e.g. $\mathrm{CH}_{3}$ or $\mathrm{CF}_{3}$ ) of aliphatic SAMs/Au(111). ${ }^{[4-6]}$ Thus, a possibility of using low-energy molecular ion beams for analysis of the molecule-vacuum interface in SAMs was clearly demonstrated.

More recently, aromatic thiols have moved into the focus of interest mainly due to their potential use in molecular electronics. ${ }^{[7-9]}$ However, the stress which originates from the misfit between the structure preferred by the aromatic moieties and the structural template provided by the $\mathrm{Au}(111)$ substrate usually results in higher defect concentration in SAMs of aromatic thiols on $\mathrm{Au}(111) \cdot{ }^{[10,11]}$ One way to overcome this problem can be realized by introducing an alkane spacer chain between the thiol head group and the aromatic moiety, as demonstrated in previous studies on the model system of biphenyl-substituted alkanethiols BPnS $\left[\mathrm{CH}_{3}-\left(\mathrm{C}_{6} \mathrm{H}_{4}\right)_{2}-\left(\mathrm{CH}_{2}\right)_{n}-\mathrm{SH}, \quad n=1-6\right]$ on a $\mathrm{Au}(111)$ substrate. ${ }^{[10]} \mathrm{By}$ addition of the alkane spacer the individual thiolates forming these SAMs have additional degrees of freedom through which stress is reduced without breaking up the film structure. However, insertion of the flexible alkane spacer also exerts a crucial influence on the molecular orientation and packing, and has much deeper consequences on the film structure and stability. Earlier microscopic ${ }^{[12,13]}$ and spectroscopic $^{[14,15]}$ studies revealed that BPnS/Au(111) SAMs exhibit a pronounced alternation in molecular structure between $n=$ odd and $n=$ even. As a result the odd-numbered BPnS/Au(111)

[a] Dr. F. Vervaecke, Dr. S. Wyczawska, Dr. J. Bastiaansen, Prof. R. E. Silverans, Dr. E. Vandeweert, Prof. P. Lievens

Laboratory of Solid State Physics and Magnetism K.U. Leuven

Celestijnenlaan 200D, 3001 Leuven, Belgium

Fax: $(+32) 16327983$

E-mail:Peter.Lievens@fys.kuleuven.be

[b] Dr. P. Cyganik, Prof. Z. Postawa

Smoluchowski Institute of Physics

Jagiellonian University

Reymonta 4, 30-059 Krakow, Poland

Fax: $(+48) 126337086$

E-mail:piotr.cyganik@uj.edu.pl
SAMs are characterized by higher density structures, with the BPnS molecules in a more upright orientation, as compared to even-numbered systems (Figure 1). Importantly, this odd-even

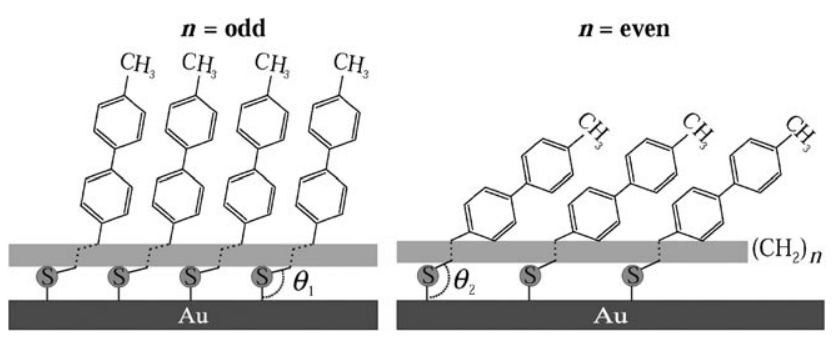

Figure 1. Schematic drawing of the orientation and the packing of BPnS molecules adsorbed on $\mathrm{Au}(111)$ for an odd and even number of methylene units in the alkyl part (parameter $n$ ). $\theta$ marks the C-S-Au angle. $\theta_{1}$ and $\theta_{2}$ are about $104^{\circ}$ and $130^{\circ}$, respectively.

variation in the $\mathrm{BPnS} / \mathrm{Au}(111)$ film structure is also reflected in different aspects of the film stability such as: 1) stability against exchange by other thiols, ${ }^{[16,17]}$ 2) electrochemical stability, ${ }^{[18,19]}$ 3) stability towards electron-induced modification, ${ }^{[20]}$ and 4) stability towards thermally induced phase transitions. ${ }^{[21-23]}$ In all these odd-even effects, related to the different aspects of the film stability, the odd numbered BPnS/Au(111) SAMs are more stable. A simple phenomenological model has been proposed $^{[22]}$ for explaining this difference in stability of odd- and even-numbered BPnS/Au(111) SAMs. In this model the stability is assumed to depend on how various factors, such as intermolecular interactions, coverage, and bonding configuration at the Au-S interface, enter into the energy balance of a SAM. Here the latter factor, that is, the bonding configuration at the $\mathrm{Au}-\mathrm{S}$ interface, contributes significantly to the energetics of the SAM system. The stability of the system depends on the fact whether this factor can be optimized along with the other two factors (cooperatively) or at the expense of these factors (competitively). The cooperative way leading to a stable system is realized in odd-numbered BPnS/Au(111) SAMs. In these systems the high coverage structure and minimized intermolecular interactions can be achieved simultaneously with the optimized $\mathrm{Au}-\mathrm{S}$ bonding configuration defined by the $\mathrm{Au}-\mathrm{S}-\mathrm{C}$ bond angle (see angle $\theta$ in Figure 1 ) and the sulphur adsorption sites. The opposite situation occurs for the evennumbered $\mathrm{BPnS} / \mathrm{Au}(111)$ SAMs where competition between optimization of the $\mathrm{Au}-\mathrm{S}$ bonding configuration and the other two factors leads to the formation of the structure with lower stability. 
The key assumption of the above model, ${ }^{[22]}$ namely that the significant modification of the Au-S interface energetics for odd- and even-numbered BPnS/Au(111) triggers the observed odd-even structural effect, was hitherto hypothetical and has not been verified experimentally so far. Herein, in order to verify this key assumption, we report on the analysis of ion-induced desorption of $\mathrm{BPnS} / \mathrm{Au}(111)$ with secondary neutral mass spectrometry (SNMS) as a new approach to probe the strength of the molecule-substrate interaction in SAMs.

Indeed, earlier experiments have indicated high sensitivity of the ion-induced desorption process to minor changes in the SAM structure. ${ }^{[24,25]}$ The bombardment of aromatic thiol SAMs on a $\mathrm{Au}(111)$ substrate with beams of $\mathrm{Ar}^{+}$ions in the keV range results in the ejection of neutral molecular fragments via two distinct desorption mechanisms. ${ }^{[24,26-29]}$ A small percentage of the SAM fragments is desorbed with high kinetic energies $(\sim \mathrm{eV})$ as a result of the direct momentum transfer from the collision cascade that develops in the substrate upon primary ion impact. The majority of desorbing particles, however, leaves the surface with low kinetic energies $\left(\sim 10^{-2} \mathrm{eV}\right)$ as a result of gentle (i.e. without significant momentum transfer) cleavage of chemical bonds within the organic layer by chemical reactions initiated by reactive fragments (e.g. $\mathrm{H} \cdot$ ) created following the impact of the primary ion. Earlier ion-induced desorption experiments performed for aromatic SAMs which form different structural phases on the $\mathrm{Au}(111)$ surface, unambiguously demonstrated that the ion-induced cleavage of chemical bonds in SAMs is extremely sensitive to details of their geometric and electronic configuration, due to the chemical reaction mechanisms involved. ${ }^{[25]}$ Here we apply this approach to probe the stability of the molecule-substrate interface of BPnS/Au(111) SAMs as a function of the chain length parameter $n$.

Figure 2 shows the mass spectra of photoionized neutral particles emitted with low kinetic energies $\left(\sim 10^{-2} \mathrm{eV}\right)$ following $15 \mathrm{keV} \mathrm{Ar}^{+}$ion beam irradiation of SAMs consisting of BPnS molecules adsorbed on the $\mathrm{Au}(111)$ substrate. A closer inspection of the data, shown in Figure 2, reveals several molecular fragments that are common for all BPnS/Au(111) mass spectra. These characteristic fragments are indicated in Figure 3 . The first characteristic fragment is at an $\mathrm{m} / \mathrm{z}$ of 168 and corresponds to the tail group containing two phenyl rings and one methylene group. The next common fragment is located at an $\mathrm{m} / \mathrm{z}$ of 181 and corresponds to the same tail group but with an additional methylene unit. The other two fragments, indicated in Figure 2, correspond to the desulphurized molecule $(\mathrm{BPnS}-\mathrm{S})$ and the intact parent molecule (BPnS), respectively. Fragments with an $m / z$ value between 181 and the value for the respective desulphurized molecule (BPnS - S), differ from each other by a single methylene unit $\left(\mathrm{CH}_{2}\right)$. The cleavage of chemical bonds in the self-assembled monolayer occurs upon the impact of energetic ions. However, molecular fragments can also originate from the interaction of desorbed species with the fairly intense laser light. Performing photon-fluence dependence measurements on gas-phase molecules demonstrated that the fragment $\mathrm{m} / \mathrm{z} 168$, the desulphurized molecule $(\mathrm{BPnS}-\mathrm{S})$, and the parent molecule $(\mathrm{BPnS})$ are direct products

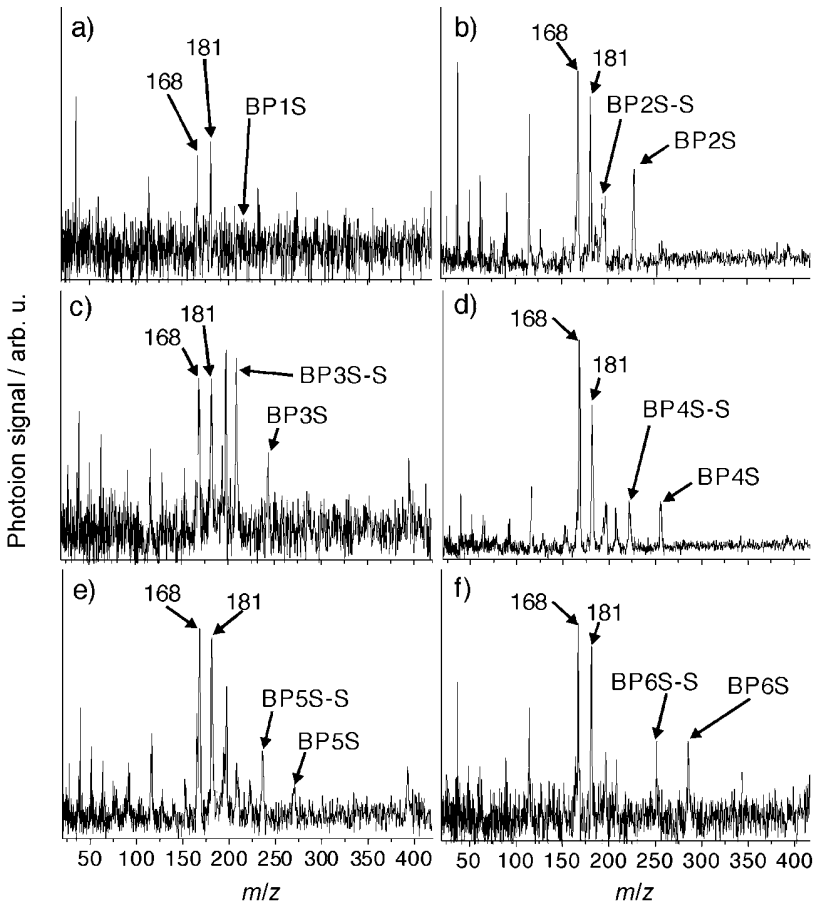

Figure 2. Mass spectra obtained from photoionization of low kinetic energy neutral molecular fragments desorbed during the $\mathrm{Ar}+$ irradiation of BPnS/ Au(111) SAMs. a) $n=1$, b) $n=2$, c) $n=3$, d) $n=4$, e) $n=5$, f) $n=6$.

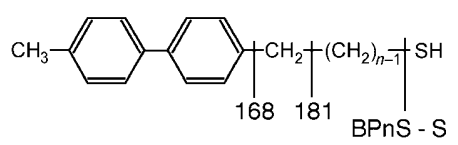

Figure 3. BPnS molecule with indicated fragments corresponding to $\mathrm{m} / \mathrm{z} 168$ and 181, the desulphurized fragment (BPnS - S) and the parent molecule (BPnS), respectively.

of the ion-induced desorption process, while the other molecular fragments, including $\mathrm{m} / \mathrm{z} 181$, at least partly stem from photofragmentation of larger molecular entities. ${ }^{[24,25,29]}$

A normalization of the mass spectra is necessary in order to correct for small experimental variations between the different sets of measurements. For normalization we use the total detected amount of desorbed molecular material with low kinetic energy. The total yield as a function of the number of $\mathrm{CH}_{2}$ units in the alkane spacer is presented in Figure 4 and shows a pronounced zigzag-like variation with respect to the odd and even number of methylene units in the BPnS molecule. Similar behavior also is found in the data shown in Figure 5, which summarizes the relative abundances of the ion signal corresponding to the tail fragment with $\mathrm{m} / \mathrm{z} 168$ (Figure $5 \mathrm{a}$ ), the desulphurized molecular fragment (Figure $5 \mathrm{~b}$ ), and the parent molecule (Figure $5 \mathrm{c}$ ) normalized with respect to the total yield. As a result, in the case of even-numbered BPnS/Au monolayers, the relative abundance of the tail fragment as well as the parent molecule is larger than for odd-numbered BPnS/Au monolayers. This odd-even effect resembles the dependence of the total molecular yield plotted in Figure 4. In contrast, the dependence of the normalized abundance of the emitted de- 


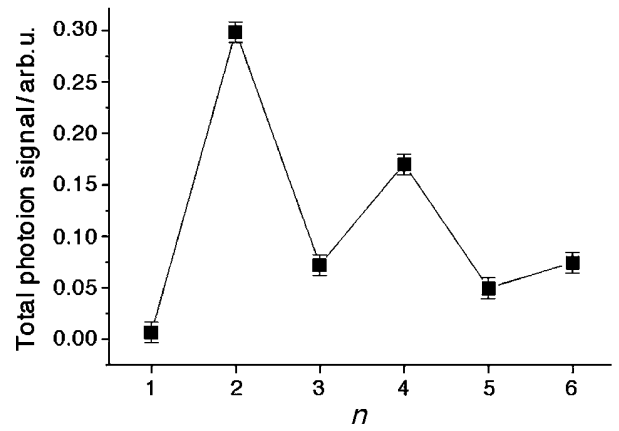

Figure 4. The total yield of molecular material desorbed with low kinetic energy from $\mathrm{BPnS} / \mathrm{Au}(111) \mathrm{SAMs}$ during $15 \mathrm{keV} \mathrm{Ar}^{+}$ion irradiation as a function of the number of methylene units (parameter $n$ ) in the alkane spacer of the BPnS molecules.
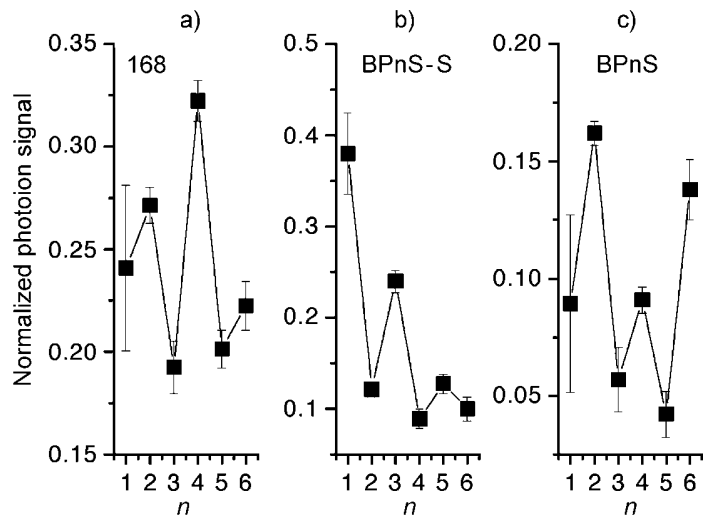

Figure 5. The photoion signal corresponding to a) the tail fragment with mass 168 amu, b) the desulphurized molecular fragment, and c) the parent molecules, normalized to the total amount of detected organic material desorbed during the $\mathrm{Ar}^{+}$irradiation of $\mathrm{BPnS} / \mathrm{Au}$. The signals are plotted as function of the number of methylene units (parameter $n$ ) in the alkane spacer of the BPnS molecules.

sulphurized molecules shows the opposite behavior, that is, the desulphurized fragments are desorbed preferentially from the odd-numbered BPnS/Au.

Importantly, desorption of the complete molecule (BPnS), the desulphurized fragment (BPnS - S), and the tail fragment $(m / z=168)$ requires the scission of a particular chemical bond, namely, $\mathrm{Au}-\mathrm{S}, \mathrm{S}-\mathrm{C}$, and $\mathrm{C}-\mathrm{C}$, respectively. Thus, results summarized in Figure 5 show that the resistance of these bonds towards the ion-induced desorption is affected by the oddeven changes in the length of the aliphatic linker. Since the desorption events analyzed in our studies (only the low-energy fragments are taken into account) can be ascribed to ion-induced chemical reactions, we suppose that the here observed lower desorption efficiency of complete molecules for odd numbered systems is due to, apparently, higher stability of the $\mathrm{Au}-\mathrm{S}$ bond in odd-numbered systems. The stability of the molecule-substrate binding in BPnS/Au(111) SAMs is crucial for their electrochemical desorption ${ }^{[18,19]}$ and exchang $\mathrm{e}^{[16,17]}$ by alkanethiols. Therefore, higher stability of odd-numbered systems with respect to these processes reported in previous ex- periments supports our conclusion that the $\mathrm{Au}-\mathrm{S}$ bond is more stable for odd-numbered systems.

The odd-even effect in the S-C bond scission, observed by desorption of the desulphurized fragment (BPnS - S), is in antiphase when compared to the $\mathrm{Au}-\mathrm{S}$ bond scission discussed above (see Figure 5). This is an important observation since one would rather expect the phase of the odd-even effect observed in the desorption efficiency of individual species to follow the phase of the odd-even effect in the total desorption yield, as it is observed for the Au-S bond scission. Following the argumentation we have used above to discuss the oddeven effect in the $\mathrm{Au}-\mathrm{S}$ bond, analoguous data obtained for the $\mathrm{S}-\mathrm{C}$ bond scission indicate that this bond is more stable for even-numbered BPnS/Au(111) SAMs. As a result we conclude that the odd systems are characterized by a more stable $\mathrm{Au}-\mathrm{S}$ bond and at the same time a less stable $\mathrm{S}-\mathrm{C}$ bond as compared to even systems. Since both bonds involve the same sulphur atom, it is rather expected that stronger bonding of the sulphur to the gold atom (or gold atoms, depending on the exact binding configuration of $S$ on a $A u(111)$ surface) will weaken its bonding to the carbon atom in the $\mathrm{Au}-\mathrm{S}-\mathrm{C}$ interface. Thus, the here observed antiphase relation in the Au-S and $\mathrm{S}-\mathrm{C}$ bond scission is fully consistent with our hypothesis.

Although performed with some simplifications with respect to the $A u(111)$ substrate reconstruction and the exact form of the unit cell for even-numbered systems, recent density functional theory (DFT) calculations suggest that odd and even $\mathrm{BPnS} / \mathrm{Au}(111)$ SAMs differ in the sulphur adsorption site and the $\mathrm{Au}-\mathrm{S}-\mathrm{C}$ bond angle. ${ }^{[30]}$ Moreover, these DFT calculations also predict that the odd-even effect is indeed a consequence of the cooperative and competitive contribution of the $\mathrm{Au}-\mathrm{S}-\mathrm{C}$ bond configuration to the energetics of the BPnS/Au(111) system. Thus, our conclusion that the stability of the Au-S and $\mathrm{S}-\mathrm{C}$ bond are different for odd and even BPnS/Au(111) systems, respectively, also is in line with the available theoretical data.

The odd-even effect is also visible in the scission efficiency of the $\mathrm{C}-\mathrm{C}$ bond that links two separate parts of the molecular backbone, that is, the alkane spacer and the biphenyl tail, resulting in a higher emission of the $m / z=168$ fragment for even numbered BPnS/Au(111) SAMs. The stability of this $\mathrm{C}-\mathrm{C}$ bond should be affected by the relative conformation of these two separate parts of the molecular backbone. Since previous spectroscopic studies ${ }^{[15]}$ demonstrated a different orientation of the biphenyl part for odd- and even-numbered BPnS/ $\mathrm{Au}(111) \mathrm{SAMs}$, the here observed odd-even effect in the stability of this $\mathrm{C}-\mathrm{C}$ bond is consistent with the odd-even variations in the BPnS/Au(111) film structure.

Besides a clear odd-even effect in the total desorption signal we also observe that with increasing chain length the desorption efficiency for even-numbered BPnS/Au(111) SAMs gradually approaches the value which is typical for odd-numbered systems (see Figure 4). Interestingly, the same type of behaviour was observed in recent experiments analyzing the stability of $\mathrm{BPnS} / \mathrm{Au}(111) \mathrm{SAMs}$ towards exchange by alkanethiols, that is, with increasing parameter $n$ the stability of the even systems is approaching the stability of the odd systems. ${ }^{[17]}$ In both experi- 
ments such behavior can be understood by considering that an increased length of the aliphatic chain gives more options to accommodate the unfavorable orientation of the biphenyl moiety in even-numbered BPnS SAMs, and thus, should gradually bring the stability of these systems towards the level of odd-numbered BPnS SAMs.

It also should be noted that the here observed chain length effect in BPnS/Au(111) SAMs differs significantly from earlier secondary ion mass spectrometry (SIMS) studies of alkanethiol SAMs on $\mathrm{Au}(111))^{[31]}$ There, a logarithmic decay in emission of all characteristic molecular ions with an increased length of the chain was observed, however, without odd-even effects. Indeed, in the case of alkanethiols/Au(111) only the conformation of the terminal methyl group is influenced by the oddeven length of the chain, while neither the molecular packing density nor the $\mathrm{Au}-\mathrm{S}-\mathrm{C}$ interface are affected.

In summary, the reported experiments clearly indicate that odd-even variations in the $\mathrm{BPnS} / \mathrm{Au}(111)$ film structure are associated with significant modification of the $\mathrm{Au}-\mathrm{S}$ bond stability at the molecule-substrate interface. We conclude that odd numbered $\mathrm{BPnS} / \mathrm{Au}(111)$ SAMs are characterized by a more stable $\mathrm{Au}-\mathrm{S}$ bond as compared to the even numbered systems. This experimental finding confirms the assumption of an earlier qualitative mode ${ }^{[22]}$ ascribing odd-even variation in the $\mathrm{BPnS} / \mathrm{Au}(111)$ stability to significant modifications at the molecule-substrate interface. Moreover, the results obtained directly indicate the nature of these modifications as due to the systematic changes in the stability of $\mathrm{Au}-\mathrm{S}$ and $\mathrm{S}-\mathrm{C}$ bonds.

In addition, the present experiments also show that the combination of ion-induced desorption and resonance enhanced ionization mass spectrometry manifests itself as a powerful technique, providing insight in the stability of the molecule-substrate interface in the SAMs.

\section{Experimental Section}

SAMs were formed by immersing gold-coated mica substrates ${ }^{[32]}$ in a $0.1 \mathrm{~mm}$ ethanolic solution of BPnS $(n=1-6)$ for at least $24 \mathrm{~h}$. The synthesis of molecules is described elsewhere. ${ }^{[15]}$ Detailed information about the setup is available elsewhere. ${ }^{[33]}$ In brief, the desorption was induced by a pulsed ( $500 \mathrm{~ns}) \mathrm{Ar}^{+}$ion beam ( $15 \mathrm{keV}$ ) directed onto the sample surface at an incidence angle of $45^{\circ}$. The experiments were performed in the static regime with a total ion fluence of about $8 \times 10^{10}$ ions $\mathrm{cm}^{-2}$. Laser postionization in combination with mass spectrometry was applied to ionize and detect neutral molecular fragments. The cloud of desorbed particles was intersected at a fixed distance of $4 \mathrm{~mm}$ parallel to the sample surface by a focused, pulsed $(6 \mathrm{~ns}, 10 \mathrm{~Hz})$ laser beam. In order to reduce the photofragmentation process, resonance enhanced multi-photon ionization (REMPI) was used. To ionize the phenylcontaining molecular particles, resonant two-photon one-colour photoionization was used at $259 \mathrm{~nm}$ with relatively low photon fluence $\left(\sim 10^{18}\right.$ photons $\left.\mathrm{cm}^{-2}\right){ }^{[34]}$ The created photoions were subsequently accelerated and detected in a linear time-of-flight mass spectrometer with a mass resolution of about 200. Changing the delay time between the incidence of the primary ion beam pulse on the sample and the firing moment of the laser, kept at a fixed position above the sample surface, enables selection of the kinetic energy of the analyzed species. To acquire mass spectra which result from ion-induced desorption by chemical reactions mechanism, only low-energy molecular species where analyzed in the present experiments. Note that not all observed molecular fragments exactly correspond to a given $\mathrm{m} / \mathrm{z}$ value. We attribute the observed spread to the capture or loss of one or several protons during the desorption and/or the photoionization processes.

\section{Acknowledgements}

The authors thank Dr Manfred Buck from St. Andrews University for providing the BPnS molecules. This work is financially supported by the Research Foundation Flanders (FWO), the Flemish Concerted Action (GOA), the Interuniversity Poles of Attraction (IAP) Research Programs, and by the Polish Ministry of Science and Higher Education (0061/B/H03/2008/34). P.C. acknowledges support received from the Foundation for Polish Science under Homing fellowship.

Keywords: ion desorption - laser postionization - odd-even effect $\cdot$ self-assembled monolayers $\cdot$ snms

[1] J. C. Love, L. A. Estroff, J. K. Kriebel, R. G. Nuzzo, G. M. Whitesides, Chem Rev. 2005, 105, 1103-1170.

[2] C. Vericat, M. E. Vela, G. A. Benitez, J. A. Martin Gago, X. Torrelles, R. C. Salvarezza, J. Phys. Condens. Matter 2006, 18, R867-R900.

[3] F. Tao, S. L. Bernasek, Chem. Rev. 2007, 107, 1408-1453.

[4] K. V. Wolf, D. A. Cole, S. L. Bernasek, Langmuir 2001, 17, 8254-8259.

[5] V. J. Angelico, S. A. Mitchell, V. H. Wysocki, Anal. Chem. 2000, 72, $2603-$ 2608.

[6] D. L. Smith, V. H. Wysocki, R. Colorado, O. E. Shmakova, M. Kraupe, T. R. Lee, Langmuir 2002, 18, 3895-3902.

[7] J. P. Hong, A. Y. Park, S. Lee, J. Kang, N. Shin, D. Y. Yoon, Appl. Phys. Lett. 2008, 92, 143311.

[8] C. Li, D. H. Zhang, X. L. Liu, S. Han, T. Tang, C. W Zhou, W. Fan, J. Koehne, J. Han, M. Meyyappan, A. M. Rawlett, D. W. Price, J. M. Tour, Appl. Phys. Lett. 2003, 82, 645-647.

[9] A. J. Kronemeijer, H. B. Akkerman, T. Kudernac, B. J. van Wees, B. L. Feringa, P. W. M. Blom, B. de Boer, Adv. Mater. 2008, 20, 1467-1473.

[10] P. Cyganik, M. Buck, J. D. E. Wilton-Ely, C. Wöll, J. Phys. Chem. B 2005, 109, $10902-10908$.

[11] D. Käfer, G. Witte, P. Cyganik, A. Terfort, C. Wöll, J. Am. Chem. Soc. 2006, $128,1723-1732$.

[12] P. Cyganik, M. Buck, W. Azzam, C. Wöll, J. Phys. Chem. B 2004, 108, 4989-4969.

[13] W. Azzam, P. Cyganik, G. Witte, M. Buck, C. Wöll, Langmuir 2003, 19, $8262-8270$

[14] K. Heister, H. T. Rong, M. Buck, M. Zharnikov, M. Grunze, L. S. O. Johansson, J. Phys. Chem. B 2001, 105, 6888-6894.

[15] H. T. Rong, S. Frey, Y. J. Yang, M. Zharnikov, M. Buck, M. Wühn, C. Wöll, G. Helmchen, Langmuir 2001, 17, 1582-1593.

[16] T. Felgenhauer, H. T. Rong, M. Buck, J. Electroanal. Chem. 2003, 550, $309-319$.

[17] K. Szelagowska-Kunstman, P. Cyganik, B. Szüpbach, A. Terfort, Phys. Chem. Chem. Phys. 2010, 12, 4400-4406.

[18] I. Thom, M. Buck, Surf. Sci. 2005, 581, 33-46.

[19] Y. T. Long, H. T. Rong, M. Buck, M. Grunze, J. Electroanal. Chem. 2002 $524,62-67$.

[20] S. Frey, H. T. Rong, K. Heister, Y. J. Yang, M. Buck, M. Zharnikov, Langmuir 2002, 18, 3142-3150

[21] P. Cyganik, M. Buck, J. Am. Chem. Soc. 2004, 126, 5960-5961.

[22] P. Cyganik, M. Buck, T. Strunskus, A. Shaporenko, J. D. E. Wilton-Ely, M. Zharnikov, C. Wöll, J. Am. Chem. Sci. 2006, 128, 13868-13878.

[23] P. Cyganik, M. Buck, T. Strunskus, A. Shaporenko, G. Witte, M. Zharnikov C. Wöll, J. Phys. Chem. C 2007, 111, 16909-16919. 
[24] P. Cyganik, E. Vandeweert, Z. Postawa, J. Bastiaansen, F. Vervaecke, P. Lievens, R. E. Silverans, N. Winograd, J. Phys. Chem. B 2005, 109, $5085-$ 5094.

[25] F. Vervaecke, S. Wyczawska, P. Cyganik, Z. Postawa, M. Buck, R. E. Silverans, P. Lievens, E. Vandeweert, J. Phys. Chem. C 2008, 112, 2248-2251.

[26] D. E. Riederer, R. Chatterjee, S. W. Rosencrance, Z. Postawa, T. D. Dunbar, D. L. Allara, N. Winograd, J. Am. Chem. Soc. 1997, 119, 8089-8094.

[27] P. Cyganik, Z. Postawa, C. A. Meserole, E. Vandeweert, N. Winograd, Nucl. Instrum. Methods Phys. Res. Sect. B 1999, 148, 137-142.

[28] Z. Postawa, C. A. Meserole, P. Cyganik, J. Szymonska, N. Winograd, Nucl. Instrum. Methods Phys. Res. Sect. B 2001, 182, 148-154.

[29] E. Vandeweert, J. Bastiaansen, F. Vervaecke, P. Lievens, R. E. Silverans, P. Cyganik, Z. Postawa, H. T. Rong, M. Buck, Appl. Phys. Lett. 2003, 82, $1114-1116$.

[30] G. Heimel, L. Romaner, J. Bredas, E. Zojer, Langmuir 2008, 24, 474-482.
[31] K. V. Wolf, D. A. Cole, S. L. Bernasek, J. Phys. Chem. B 2002, 106, 10382 10387.

[32] N. Vandamme, G. Verschoren, A. Depuydt, M. Cannaerts, W. Bouwen, P. Lievens, R. E. Silverans, C. Van Haesendonck, Appl. Phys. A 2001, 72, S177.

[33] E. Vandeweert, P. Lievens, V. Philipsen, J. Bastiaansen, R. E. Silverans, Phys. Rev. B 2001, 64, 195417.

[34] E. Vandeweert, C. A. Meserole, A. Sosteracz, Y. Dou, N. Winograd, Z Postawa, Nucl. Instrum. Methods Phys. Res. Sect. B 2000, 164-165, $820-$ 826.

Received: July 27, 2010

Published online on December 8, 2010 